\title{
AN AXIOMATIC APPROACH TO QUANTUM GAUGE FIELD THEORY
}

\author{
THOMAS THIEMANN \\ Physics Department, Harvard University \\ Cambridge, MA 02138, U.S.A. \\ E-mail: thiemann@math.harvard.edu
}

\begin{abstract}
In the present article we display a new constructive quantum field theory approach to quantum gauge field theory, utilizing the recent progress in the integration theory on the moduli space of generalized connections modulo gauge transformations.

That is, we propose a new set of Osterwalder Schrader like axioms for the characteristic functional of a measure on the space of generalized connections modulo gauge transformations rather than for the associated Schwinger distributions.

We show non-triviality of our axioms by demonstrating that they are satisfied for twodimensional Yang-Mills theory on the plane and the cylinder.

As a side result we derive a closed and analytical expression for the vacuum expectation value of an arbitrary product of Wilson-loop functionals from which we derive the quantum theory along the Glimm and Jaffe algorithm which agrees exactly with the one as obtained by canonical methods.
\end{abstract}

1. Introduction. It is an old dream of theoretical physicists to base the description of Yang-Mills (gauge) theories on the so-called Wilson-loop observables. These are simply (products of) traces of holonomies around closed loops in the given spacetime. Also general relativity, when formulated as a dynamical theory of connections, can be explored via Wilson-loop variables [1].

The advantage of these observables is that they provide for an overcomplete set of coordinates for the gauge invariant information that is contained in the connection [8], also called the moduli space of (spacetime) connections modulo gauge transformations, $\mathcal{A} / \mathcal{G}$. There are two major disadvantages:

1) The space $\mathcal{A} / \mathcal{G}$ is nonlinear. Therefore, all the mathematical physics techniques that have been developed for field theories whose underlying space of fields is linear are not available. A solution out of this is to fix a gauge and to work with Schwinger functions of

1991 Mathematics Subject Classification: 81T08, 28C05, 28C10, 28C15, 28C20, 60Gxx, 81-xx. The paper is in final form and no version of it will be published elsewhere. 
connections in that gauge but that comes at a price: manifest gauge invariance is lost and we have the problem of the annoying Gribov copies. Also from a geometrical viewpoint it just does not seem to be right to enforce linearity by brute force.

2) If one keeps manifest gauge invariance, and the only way to do this as far as we know is to work with Wilson-loop functionals ${ }^{1}$, then the connection is smeared with a loop function. There is then an immediate problem: it is well-known that linear quantum fields are rather distributional and need to be smeared in all spacetime directions, however, a loop only smears in one such direction. That either means that we have to give up this approach or that YM quantum fields are simply better behaved in the precise sense that the (vacuum expectation value of the) Wilson observables exists. There is a chance that this is true, at least in the non-Abelian case, since the physically relevant phase of, say, QCD is not described by the Fock Hilbert space.

In this article we take advantage of the existence of new integration techniques developed in $[3,2,14]$ in order to set up a system of Osterwalder-Schrader (OS) axioms that are tailored to $\mathcal{A} / \mathcal{G}$ [4]. Our axioms are imposed directly on the measure rather than on the associated Schwinger distributions [17] and is thus more in the fashion of [9]. This will enable us to circumvent all the problems that are connected with these earlier approaches. Our approach is as rigorous as the ones in [9] for the linear case or [17] for the YM case. Furthermore, we prove non-triviality of these axioms by showing that they have a non-trivial solution, namely we verify them for two-dimensional pure YM theory for any semi-simple compact gauge group which is known to be an integrable, finite dimensional model.

The paper is organized as follows:

In Section 2 we review the relevant notions from calculus on $\mathcal{A} / \mathcal{G}$.

In Section 3 we motivate and introduce a new set of axioms tailored to quantum gauge field theory.

In Section 4 we derive the general form for the generating functional of the YangMills measure on $\mathcal{A} / \mathcal{G}$ for any compact semi-simple gauge group for the two-dimensional spacetimes of the topology of the plane and the cylinder which are the ones of physical relevance.

In Section 5 we explicitly verify the new axioms for the model analysed in Section 4 and give the relation to the Hamiltonian approach.

\section{Preliminaries.}

\subsection{Integration on $\mathcal{A} / \mathcal{G}$.}

We review here the necessary notions from $[3,2,14,4]$ and references therein.

We will consider the set $\mathcal{L}_{p}$ of oriented unparametrized loops based at an arbitrary but fixed point $p$ of spacetime $M$ as the entity of piecewise analytical embeddings of the circle into $M$. Throughout this paper we will deal only with based, piecewise analytical loops. With respect to the natural composition of loops, $\mathcal{L}_{p}$ adopts the structure of a semi-group.

\footnotetext{
${ }^{1}$ for instance, invariants constructed from the curvature suffer from the field copy problem $[20,21]$.
} 
With the help of the space $\mathcal{A} / \mathcal{G}$ of smooth connections modulo gauge transformations we turn $\mathcal{L}_{p}$ into a group $\mathcal{H} \mathcal{G}$, called the hoop group in the sequel, as follows:

We define two loops $\alpha_{1}, \alpha_{2} \in \mathcal{L}_{p}$ to be holonomically equivalent, $\alpha_{1} \sim \alpha_{2}$, iff their holonomies agree on every point $A \in \mathcal{A} / \mathcal{G}$, that is, $h_{\alpha_{1}}(A)=h_{\alpha_{2}}(A) \forall A \in \mathcal{A} / \mathcal{G}$. Here the holonomy map is defined as the path-ordered exponential of the line integral of the connection along the loop:

$$
h_{\alpha}(A):=\mathcal{P} \exp \left(\oint_{\alpha} A\right) .
$$

The symbol $\mathcal{P}$ asks that in any parametrization of the loop the terms with the highest values of the parameter be ordered to the left. Then $\mathcal{H G}:=\mathcal{L}_{p} / \sim$. We will not distinguish any more in the sequel between a hoop and its various representants and will use the word loop again unless confusion could arise.

We will assume once and for all that the gauge group $G$ is compact and semisimple of rank $r$ (the non-semisimple case can be treated in a similar manner). The associated principal fibre bundle is taken to be trivial.

We now introduce the so-called Wilson-loop functionals [8]

$$
T_{\alpha}(A):=\frac{1}{N} \operatorname{tr}\left(h_{\alpha}(A)\right)
$$

where the trace is taken with respect to the $N$-dimensional fundamental representation of $G$. The Wilson loops are manifestly gauge invariant functions on $\mathcal{A} / \mathcal{G}$ and are separating on $\mathcal{A} / \mathcal{G}$ in the sense that given all the $T_{\alpha}$, we can reconstruct the smooth connection modulo gauge transformations [8].

These quantities enable us to construct an Abelian $C^{*}$-algebra as follows: consider the quantities of the form

$$
\Psi:=\sum_{I=1}^{r} \sum_{i=1}^{n} z_{I i} \prod_{J=1}^{I} T_{\alpha_{I i, J}} .
$$

The system of these objects is easily checked to be an Abelian algebra: the Mandelstam identities [8] reveal that every product of traces of the holonomy can be written as a linear combination of products of Wilson loops with at most $r$ factors. Moreover, it is an Abelian *-algebra since $\bar{T}_{\alpha}=T_{\alpha^{-1}}$. Finally, we turn it into an Abelian $C^{*}$-algebra by completing it with respect to the norm

$$
\|\Psi\|:=\sup _{A \in \mathcal{A} / \mathcal{G}}|\Psi|(A) .
$$

The $C^{*}$ property follows easily from that for complex numbers.

This Abelian $C^{*}$-algebra will be called the holonomy algebra $\mathcal{H} \mathcal{A}$.

We can now employ usual Gel'fand theory: The Gel'fand spectrum $\overline{\mathcal{A} / \mathcal{G}}$ of generalized connections is in one-to-one correspondence with the space of all homomorphisms from $\mathcal{H G}$ into the gauge group $G$, that is to say, it is the algebraic dual of $\mathcal{H G}$. By the RieszMarkov theorem, regular Borel measures on $\overline{\mathcal{A} / \mathcal{G}}$ are in one-to-one correspondence with positive linear functionals on the space of continuous functions on $\overline{\mathcal{A} / \mathcal{G}}$ (recall that with respect to the Gel'fand topology $\overline{\mathcal{A} / \mathcal{G}}$ is a compact Hausdorff space).

An interesting example of a measure $\mu_{0}$ on $\overline{\mathcal{A} / \mathcal{G}}$ has been constructed in [3]: 
Consider the family of all piecewise analytical oriented graphs $\Gamma$ in $M$, that is, piecewise analytic embeddings of closed intervals in $M$. We will denote its fundamental group by $\pi_{1}(\Gamma)$. Choose a system of generators $\beta_{1}, \ldots, \beta_{n}$ of $\pi_{1}(\Gamma)$ where $n:=d_{\Gamma}:=\operatorname{dim}\left(\pi_{1}(\Gamma)\right)$ is the dimension of the fundamental group. A cylindrical function $f$ on $\overline{\mathcal{A} / \mathcal{G}}$ can be written as the pull-back under the following map for one of the graphs $\Gamma$ :

$$
p_{\Gamma}: \overline{\mathcal{A} / \mathcal{G}} \rightarrow G^{d_{\Gamma}} ; \quad A \mapsto\left(h_{\beta_{1}}(A), \ldots, h_{\beta_{d_{\Gamma}}}(A)\right),
$$

that is, $f=\left(p_{\Gamma}\right)^{*} f_{\Gamma}$ where $f_{\Gamma}$ is a map from $G^{d_{\Gamma}}$ into the complex numbers.

The measure $\mu_{0}$ is then defined to be the following linear functional for cylindrical functions

$$
\begin{aligned}
\int_{\overline{\mathcal{A} / \mathcal{G}}} d \mu_{0}(A) f(A) & :=\int_{\overline{\mathcal{A} / \mathcal{G}}} d \mu_{\Gamma}(A) f_{\Gamma}\left(p_{\Gamma}(A)\right) \\
& :=\int_{G^{d}{ }_{\Gamma}} d \mu_{H}\left(g_{1}\right) \ldots d \mu_{H}\left(g_{d_{\Gamma}}\right) f_{\Gamma}\left(g_{1}, \ldots, g_{d_{\Gamma}}\right) .
\end{aligned}
$$

That this defines indeed an infinite dimensional ( $\sigma$-additive) measure $\mu_{0}$ as the projective limit [22] of the measures $\mu_{\Gamma}$ defined in (2.5) was shown in [14].

The rigorously defined measure $\mu_{0}$ will be used in the next section to construct the Yang-Mills measure.

Here are two more definitions which prove useful in the sequel.

Definition 2.1. A loop network state on a given graph $\Gamma$ with fundamental group $\left[\beta_{1}, \ldots, \beta_{n}\right]$ is labelled by a triple $(\Gamma, \vec{\pi}, c)$ consisting of that graph $\Gamma$, a vector of nontrivial irreducible representations $\vec{\pi}=\left[\pi_{1}, \ldots, \pi_{n}\right]$ and a contraction matrix $c$ which takes values in the projectors onto the orthogonal irreducible representations contained in the decomposition of $\bigotimes_{k=1}^{n} \pi_{k}$. It is defined by

$$
T_{\Gamma, \vec{\pi}, c}(A):=\operatorname{tr}\left[\bigotimes_{k=1}^{n} \pi_{k}\left(h_{\beta_{k}}(A)\right) \cdot c\right] .
$$

Different choices of generators of $\pi_{1}(\Gamma)$ lead to unitarily equivalent bases.

The loop network states can be seen to provide for a complete (and orthogonal with respect to $\left.\mu_{0}\right)$ basis of states for any cylindrical subspace of $C(\overline{\mathcal{A} / \mathcal{G}})$ defined by a graph $\Gamma[16,6,19]$. In particular, the multiloop states $T_{\alpha_{1}} \ldots T_{\alpha_{r}}$ can always be expressed in terms of those so that we arrive at the following definition:

Definition 2.2. The characteristic functional of a measure on $\overline{\mathcal{A} / \mathcal{G}}$ is defined by the set of expectation values of loop network states:

$$
\chi_{\mu}(\Gamma, \vec{\pi}, c):=\int_{\overline{\mathcal{A} / \mathcal{G}}} d \mu(A) T_{\Gamma, \vec{\pi}, c}(A) .
$$

3. A proposal for Constructive Quantum Gauge Field Theory. As already mentioned before, the concepts introduced in the textbook treatments [9] of constructive scalar field theory seem inadequate for nonlinear theories such as gauge field theories whose space of histories is given by $\mathcal{A} / \mathcal{G}$. The idea is to come up with new axioms that are guided by the ones for scalar field theory but take the nonlinearity of $\overline{\mathcal{A} / \mathcal{G}}$ fully into account. 
Although this idea is not completely new, related contributions [17] seem to be too strongly attached to techniques applicable to linear theories, mainly because these works are based on axioms for Schwinger distributions. From our point of view it seems much more natural to impose the axioms on the underlying measure.

The OS axioms for a QFT based on a linear space of histories can be roughly described as follows [9]:

One states everything in terms of the characteristic functional $\chi$ of a measure $\mu$ (its Fourier transform) which is required to be continuous and positive definite on (finite subspaces of) the space $\mathcal{S}$ of test functions of rapid decrease

$$
\chi(f):=\langle\exp (i \Phi[f])\rangle:=\int_{\mathcal{S}^{\prime}} d \mu(\Phi) \exp (i \Phi[f])
$$

and $\Phi[f]:=\int_{\mathbb{R}^{d+1}} d^{d+1} x \Phi(x) f(x)$ denotes the canonical pairing between distributions and test functions.

As this $\mathcal{S}$ is a nuclear space, Minlos' theorem [22] then tells us that (as already displayed in (3.1)) the measure has support on the space $\mathcal{S}^{\prime}$ of tempered distributions. It is obvious that right from the beginning everything is soaked into kinematical linearity.

In order to find the appropriate analogue of these notions for constructive gauge field theory, let us make some heuristic considerations:

The counterpart of the scalar field $\Phi$ is of course the connection. Since we are interested in a measure theoretic formulation of the theory, we now have to look for the analogue of the expression $\exp (i \Phi[f])$. Let us look for a moment at the Abelian case. Then the Wilsonloop functional is given by $T_{\alpha}=\exp (i A[\alpha])$ where we have allowed for a distributional connection and the canonical pairing between the field $A$ and the loop $\alpha$ is now given by $A[\alpha]=\oint_{\alpha} A=\int_{0}^{1} d t \dot{\alpha}^{a} A_{a}(\alpha(t))$ rather than $\Phi[f]=\int d^{d+1} x \Phi(x) f(x)$. This is an important difference: in order that this object makes sense, the connection is not allowed to be in $\mathcal{S}^{\prime}\left(\mathbb{R}^{d}\right)$ ! This immediately implies that the theory that we want to base on Wilson loops will not result in the usual Fock space, not even for Maxwell theory!

However, the formal similarity between the expressions $\exp (i \Phi[f])$ and $T_{\alpha}$ generalized to the non-Abelian case thus motivates to base the generating functional $\chi$ of a measure on $\overline{\mathcal{A} / \mathcal{G}}$ on the usage of Wilson loops. Since for a rank $r$ group products of Wilson-loop functionals can only be reduced to sums of products of at most $r$ Wilson-loop functionals, we arrive naturally at the expression (2.7) for the characteristic functional of a measure on $\overline{\mathcal{A} / \mathcal{G}}$. Accordingly, the analogue of the probes of the the field $\Phi$, namely test functions of rapid decrease, are piecewise analytic loops in Euclidean space.

The nice thing is that the precise analogue of Bochner's theorem can be argued to be the Riesz-Markov theorem: any positive linear functional $\chi$ on $C(\overline{\mathcal{A} / \mathcal{G}})$ gives rise to a regular Borel measure $\mu$ on $\overline{\mathcal{A} / \mathcal{G}}$ which is a compact Hausdorff space by construction.

What we do not have is an analogue of the Minlos theorem which is due to the fact that we did not specify any topology on the space of probes, i.e. the set of loops (see [18] for an attempt to build a nuclear topology on $\mathcal{H G}$ ). Since we will never need to specify the analogue of Schwinger distributions in what follows, we do not worry about that. Suffice it to say that our carrier space $\overline{\mathcal{A} / \mathcal{G}}$ is the maximal extension of $\mathcal{A} / \mathcal{G}$ such that Wilson-loops are still continuous (with respect to the Gel'fand topology on $\overline{\mathcal{A} / \mathcal{G}}$ ) and 
we do not mind working with that bigger space although the actual carrier of physically relevant measures maybe significantly smaller.

Let us now formulate analogues of the OS axioms [9]:

A Quantum gauge field theory is a probability measure $\chi$ on $\overline{\mathcal{A} / \mathcal{G}}$ satisfying the following axioms:

- OS-I) Analyticity. This axiom in scalar QFT ensures that Schwinger functions of all orders exist. Since we are not interested in these for gauge field theory, because they are not gauge invariant, we will drop that axiom here altogether!

- OS-II) Regularity. The regularity axiom for the scalar field prescribes some bound on the characteristic functional. Technically, it can be used to show that the measure is supported on the space of tempered distributions, rather than on those which are continuous on the test functions of compact support. This is important if we want to do things like Wick rotations of Schwinger functions to Wightman functions. Since we are not interested in that issue, we also simply drop this axiom!

- OS-III) Euclidean invariance. The action $g \cdot \Gamma$ of an element $g$ of the full Euclidean $E$ group in $d$ spacetime dimensions on a graph is just the image of the linear transformation $x \mapsto(g \cdot x)$ where $x$ is a point on $\Gamma$. The measure is required to be invariant under this action

$$
\chi(g \cdot \Gamma, \vec{\pi}, c)=\chi(\Gamma, \vec{\pi}, c) .
$$

- OS-IV) Reflection positivity. This is the most important of the axioms because it allows to reformulate the theory in terms of more familiar concepts, that is, it provides us with a notion of time, a Hilbert space, and a Hamiltonian (compare [9] for the proof of this fact which is completely insensitive to whether the space of histories is linear or not). The technicalities are as follows:

Choose an arbitrary hyperplane in $\mathbb{R}^{d}$ which we will call the time zero plane. Consider the linear span, denoted $V$, of the following functions on $\overline{\mathcal{A} / \mathcal{G}}$ of the form

$$
\Psi_{\left\{z_{I}\right\}}: \overline{\mathcal{A} / \mathcal{G}} \rightarrow \mathbb{C} ; \quad A \mapsto \sum_{I \in S} z_{I} T_{I}
$$

where each index $I$ stands for data $I=(\Gamma, \vec{\pi}, c)$ of a loop network and where $\Gamma$ is supported in the positive time half-space $\left\{x=\left(x^{0}, \vec{x}\right) \in \mathbb{R}^{d} ; x^{0}>0\right\}$ and $S$ is a finite set of indices. Furthermore, let $\Theta\left(x^{0}, \vec{x}\right)=\left(-x^{0}, \vec{x}\right)$ denote the time reflection operator $(\Theta \in E)$. Then it is required that for each $\psi, \Xi \in V$

$$
(\Psi, \Xi):=\langle\Theta \Psi, \Xi\rangle:=\int_{\overline{\mathcal{A} / \mathcal{G}}} d \mu(A) \overline{\Theta \Psi[A]} \Xi[A] \geq 0 .
$$

Reflection positivity has been verified on the (finite) lattice for YM theory [9].

- OS-V) Ergodicity. This axiom ensures the uniqueness of the vacuum (a vector invariant under the time translation subgroup of $\left.E, T(s)\left(x^{0}, \vec{x}\right)=\left(x^{0}+s, \vec{x}\right)\right)$. The requirement is that

$$
\lim _{t \rightarrow \infty} \frac{1}{t} \int_{0}^{t} d s(T(s) \Psi)\left[A_{0}\right]=\int_{\overline{\mathcal{A} / \mathcal{G}}} d \mu(A) \Psi[A]
$$


for any vector $\Psi \in L_{1}(\overline{\mathcal{A} / \mathcal{G}}, d \mu) \cap V$ and any $A_{0} \in \overline{\mathcal{A} / \mathcal{G}}$. Note that the right-hand side implies that the left-hand side does not depend on the particular choice of $A_{0}$.

\section{Euclidean YM gauge theory in two dimensions.}

4.1 The lattice regularization. Consider a special family of graphs $\Gamma\left(a, L_{x}, L_{y}\right)$ in $M$, namely finite square lattices with spacing $a$ of length $L_{x}$ and $L_{y}$ in $x$ and $y$ direction respectively with respect to the Euclidean norm of the 2-dimensional Euclidean spacetime $M$ (i.e. the spacetime metric is $g_{a b}=\delta_{a b}$ ). Thus we have introduced an IR regulator (the finite volume defined by $L_{x}$ and $L_{y}$ ) and an UV regulator (defined by the lattice spacing $a)$. We have $\left(N_{x}+1\right)\left(N_{y}+1\right)$ vertices on that finite lattice where $N_{x} a:=L_{x}, N_{y} a:=L_{y}$. An open path along an edge (link) $l$ of the lattice from the vertex $i$ to the vertex $j$ will be denoted by

$$
l=l_{i \rightarrow j}
$$

which enables us to define the plaquette loops $\square$ based at $(x, y)$ according to

$(4.2) \square_{(x, y)}:=l_{(x, y) \rightarrow(x, y+1)}^{-1} \circ l_{(x, y+1) \rightarrow(x+1, y+1)}^{-1} \circ l_{(x+1, y) \rightarrow(x+1, y+1)} \circ l_{(x, y) \rightarrow(x+1, y)}$.

That is, the plaquette loop starts at the bottom left corner and our convention is such that the coordinate directions define positive orientation. Here the coordinates $x, y$ are taken to be integers (in lattice units).

There are no boundary conditions for the plane $M=\mathbb{R}^{1} \times \mathbb{R}^{1}$ while we identify $l_{(1, y) \rightarrow(1, y+1)}$ and $l_{\left(N_{x}+1, y\right) \rightarrow\left(N_{x}+1, y+1\right)}$ on the cylinder $M=\mathbb{R}^{1} \times S^{1}$.

We will choose the basepoint $p$ to lie in the upper right corner of $\Gamma$ and we will use the following generators of $\pi_{1}(\Gamma)$ :

1) On the plane, choose an open path $\rho_{x, y}$ within $\Gamma$ from $p$ to the point $(x, y)$. Then we have the $N_{x} N_{y}$ generators

$$
\beta_{x, y}:=\beta_{\square(x, y)}:=\rho_{x, y}^{-1} \circ \square_{(x, y)} \circ \rho_{x, y} .
$$

2) On the cylinder we need apart from (4.3) one more generator "which wraps once around the cylinder". We will choose the horizontal loop "at future time infinity"

(4.4) $\beta_{x}:=l_{\left(N_{x}, N_{y}+1\right) \rightarrow\left(1, N_{y}+1\right)} \circ l_{\left(N_{x}-1, N_{y}+1\right) \rightarrow\left(N_{x}, N_{y}+1\right)} \circ \ldots \circ l_{\left(1, N_{y}+1\right) \rightarrow\left(2, N_{y}+1\right)}$.

We are now ready to define the regulated characteristic functional of the regulated $G$ Yang-Mills measure:

Consider the following cylindrical functions on $\overline{\mathcal{A} / \mathcal{G}}$, cylindrical relative to our lattice graph $\Gamma$ :

1) the exponential

$$
\exp \left(-\beta S_{\text {Wilson }}\right) \quad \text { where } S_{\text {Wilson }}(A):=\sum_{\square}\left[1-\frac{1}{N} \Re \operatorname{tr}\left(h_{\square}(A)\right)\right]
$$

is the so-called Wilson action for $G$ Yang-Mills theory [7], $h_{\square}$ is the holonomy along the based loop $\square, \Re \operatorname{tr}$ means "take the real part of the trace of" and the "inverse temperature" is given by

$$
\beta=\frac{1}{g_{0}^{2} a^{4-d}}
$$


( $d=2$ is the dimension of $M$ ) where $g_{0}=g_{0}(a)$ is the bare coupling. Here we have chosen a basis $\left\{\tau_{I}\right\}_{I=1}^{\operatorname{dim}(G)}$ for the Lie algebra $L(G)$ of $G$ such that $\operatorname{tr}\left(\tau_{I} \tau_{J}\right)=-N \delta_{I J}$.

2 ) the product of Wilson-loop functionals for any $r$ loops $\alpha_{1}, \ldots, \alpha_{r}$ embedded in $\Gamma=$ $\Gamma\left(a ; L_{x}, L_{y}\right)$ and $N$ is the dimension of the fundamental representation of $G$.

These functions are cylindrical since the loops $\alpha_{i}$ can, by definition of the fundamental group of a graph, be expressed as a particular composition of the generators of $\pi_{1}(\Gamma)$.

Thus, all these functions are measurable with respect to the measure $\mu_{0}$ introduced in Section 2 and the following definition makes sense:

$$
\begin{aligned}
\chi & \left(\alpha_{1}, \ldots, \alpha_{N-1} ; a ; L_{x}, L_{y}\right):=\left\langle T_{\alpha_{1}} \ldots T_{\alpha_{r}}\right\rangle \\
& :=\frac{1}{Z\left(a ; L_{x}, L_{y}\right)} \int_{\frac{\mathcal{A} / \mathcal{G}}{} d \mu_{0}(A) e^{-\beta S_{W \text { ilson }}(A)} T_{\alpha_{1}}(A) \ldots T_{\alpha_{r}}(A)} \\
& :=\frac{1}{Z} \prod_{\beta \in \pi_{1}(\Gamma)} \int_{G} d \mu_{H}\left(h_{\beta}\right) \exp \left(-\beta S_{\text {Wilson }}\right) \operatorname{tr}\left(\prod_{\beta \in \alpha_{1}} h_{\beta}\right) \ldots \operatorname{tr}\left(\prod_{\beta \in \alpha_{r}} h_{\beta}\right)
\end{aligned}
$$

where the notation $\beta \in \alpha_{i}$ means "composition of all those generators $\beta$ necessary to express $\alpha_{i}$ (in the specific order as defined by $\alpha_{i}$ )".

The partition function $Z=Z\left(a ; L_{x}, L_{y}\right)$ is defined through $\chi\left(p, \ldots, p ; a ; L_{x}, L_{y}\right)=1$ where $p$ is, as above, the basepoint of all the loops on the lattice (it is in particular a trivial loop).

The idea is now quite similar to related constructions in constructive quantum scalar field theory [15]. There, one integrates the regularized version of the exponential of $-\beta$ times the interaction part of the Euclidean action times $\exp (i \Phi[f])$ with the rigorously defined free (Gaussian) measure. Then one takes the thermodynamic (infinite volume) and continuum limit of the resulting expression and obtains the characteristic functional of a rigorously defined interacting theory.

In our case the role of the Gaussian measure is played by the rigorously defined, $\sigma$ additive measure $\mu_{0}$ on the universal carrier $\overline{\mathcal{A} / \mathcal{G}}$, the "regularized interaction part of the action" is played by the Wilson action and the analogue of $\exp (i \Phi[f])$ is given by the products of Wilson-loop functionals.

The reader might worry that we have changed lattice gauge field theory in the previous section [7]. One can show that this is not the case, i.e. both formulations are equivalent [5].

4.2 Expression of the Wilson loop in terms of plaquettes. By definition of the fundamental group of a graph, each of the $\alpha_{i}$ involved in the characteristic functional $\chi$ can be written as a particular composition of the generators of the graph (lattice). In this section we are going to characterize this composition.

First three definitions:

\section{DEFINITION 4.1}

i) A loop is said to be simple iff there is a holonomically equivalent loop which has no self-intersections.

ii) By the surface enclosed by a simple loop we mean the surface that is bounded by the simple loop and lies to the left as one follows the loop counterclockwise (mathematically positive direction). 
iii) Two distinct simple loops are said to be non-overlapping iff the intersection domain of the surfaces that they enclose has zero Euclidean area.

So, for example all the loops $\beta_{x, y}$ are simple since they lie in the same hoop class as the plaquette loops $\square_{(x, y)}$. Non-overlapping distinct simple loops may share whole segments whence the plaquette generators of our graph (lattice) are mutually non-overlapping.

The following two simple lemmas govern the form of the characteristic functional in two spacetime dimensions.

LEMMA 4.1. Every simple, homotopically trivial loop on $\Gamma$ can be written as a particular composition of the generators $\beta_{\square}$ whose surfaces are contained in the surface enclosed by that loop, each of them appearing once and only once.

Proof. We will do this by direct construction.

Consider a simple based loop of the form $\alpha=\rho^{-1} \circ \beta \circ \rho$ where $\rho$ is the given open path between $p$ and the starting point $q$ on the unbased loop $\beta$. Subdivide $\beta$ into columns parallel to the $y$-axis. If $q$ does not coincide yet with the vertex of the horizontal link with the lower $x$ argument on the bottom of the most right column then let $l$ be the edge of $\beta$ between $q$ and that point on $\beta$. Then we have $\alpha=(l \circ \rho)^{-1} \circ\left(l \circ \beta \circ l^{-1}\right) \circ(l \circ \rho)$ so that by appropriate redefinition of $\beta$ and $\rho$ we can always achieve that the starting point $q$ on $\alpha$ is the point mentioned above.

Let $q:=(x, y)$ and $n$ be the height of that most right column. Then the holonomy around $\alpha$ is given by ( $g$ is the product of the holonomies of those links that are not involved in the most right column)

$$
\begin{aligned}
& h_{\rho}^{-1} g h_{(x, y+n) \rightarrow(x+1, y+n)}^{-1} h_{(x+1, y+n-1) \rightarrow(x+1, y+n)} \ldots h_{(x+1, y) \rightarrow(x+1, y+1)} h_{(x, y) \rightarrow(x+1, y)} h_{\rho} \\
&= \operatorname{Ad}\left[h_{\rho}^{-1}\right]\left(g h_{(x, y+n-1) \rightarrow(x, y+n)} \ldots h_{(x, y) \rightarrow(x, y+1)}\right) \\
& \quad \operatorname{Ad}\left[\left(h_{(x, y+n-2) \rightarrow(x, y+n-1)} \ldots h_{(x, y) \rightarrow(x, y+1)} h_{\rho}\right)^{-1}\right]\left(\square_{x, y+n-1}\right) \ldots \operatorname{Ad}\left[h_{\rho}\right]\left(\square_{x, y}\right) \\
&=:\left\{\operatorname{Ad}\left[h_{\rho}^{-1}\right]\left(h_{\tilde{\beta}}\right)\right\} g_{x, y+n-1} \ldots g_{x, y}
\end{aligned}
$$

Here we have denoted by Ad the adjoint action of the group on itself, that is, $\operatorname{Ad}[g](h):=$ $g h g^{-1}$.

The curly bracket in the last line is the holonomy around $\alpha$ with the most right column removed and the lost segment, resulting from that removement, reattached. This loop we called $\rho^{-1} \circ \tilde{\beta} \circ \rho$. The remaining product involves all the based plaquette loops of the most right column, each of them appearing precisely once. We iterate like this to the left until we reach the last column. But the analogous curly bracket term as the one above for the last column is the identity.

LEMMA 4.2. Every loop can be written as a composition of simple non-overlapping loops.

Pr o of. Given a loop $\alpha$ on $\Gamma$, consider the pattern of surfaces on $M$ that its homotopically trivial part defines (the homotopically non-trivial loop $\beta_{x}$ are not overlapping with any other loop and do not enclose a surface). Take the boundaries of these surfaces as the definition of the simple non-overlapping loops $\alpha_{I}, I=1, \ldots, n$, and note that the pattern of surfaces defines a subgraph $\tilde{\Gamma}$ of $\Gamma$. Since the $\alpha_{I}$ are simple, by the preceding lemma, we can express one (call it $\beta_{I}$ ) of the $\beta_{x, y}$ contained in the surface enclosed by $\alpha_{I}$ by 
$\alpha_{I}$ and the rest of these $\beta_{x, y}$. This is possible because the corresponding transformation is non-singular (the $\beta_{x, y}$ contained in the surface enclosed by $\alpha_{I}$ appear precisely once). Since the $\alpha_{I}$ are non-overlapping, we conclude that the $\beta_{I}$ are all distinct. Since they belong to a generating system of loops on $\Gamma$ we see that we may choose $\alpha_{I}$ as generators as well. They obviously generate $\pi_{1}(\tilde{\Gamma})$.

The argument can be straightforwardly generalized to the case of a multiloop $\alpha_{1}, \ldots, \alpha_{r}$ by considering the pattern of surfaces made by the union of those $r$ loops. In this way, the choices of open paths $\rho_{\square}$ between $p$ and the plaquettes $\square$ made in (4.8) is consistent because the $\square$ appear in one and only one of the non-overlapping, simple, homotopically trivial loops. As already mentioned earlier, the Wilson action is not affected by such a choice.

Consider the case that the loop $\alpha$ contains a homotopically trivial loop $\lambda=\left[\lambda \circ \gamma^{-1}\right] \circ \gamma$. The loop in the bracket is homotopically trivial if $\gamma$ is in the same homotopy class as $\lambda$. Thus we are able to express $\alpha$ in the manner described above in terms of nonoverlapping simple homotopically trivial loops and our favourite homotopically non-trivial generators $\gamma$.

Summarizing, we have shown that

$$
\begin{gathered}
\chi\left(\alpha_{1}, \ldots, \alpha_{r}\right)=\frac{1}{Z} \prod_{\square} \int_{G} d \mu_{H}\left(g_{\square}\right) \exp \left(-\beta S_{\text {Wilson }}\right) \times \\
\begin{cases}\prod_{i=1}^{r} \operatorname{tr}\left(" \prod_{\square \in \alpha_{i}} g_{\square} "\right) & \text { on } \mathbb{R}^{2} \\
\int_{G} d \mu_{H}(g) \prod_{i=1}^{r} \operatorname{tr}\left(" \prod_{\gamma, \square \in \alpha_{i}} g_{\square} g^{\prime \prime}\right) & \text { on } \mathbb{R}^{1} \times S^{1}\end{cases}
\end{gathered}
$$

where the "" denote that one has to order the variables involved correctly and that each of the variables could occur more than once and in particular also its inverse. We have denoted by $g_{\square}$ and $g$ plaquette and homotopically non-trivial loop $(\gamma)$ integration variables. Always $\chi(0)=1$.

4.3 The general form of the generating functional. We now actually perform the lattice integration of any product of Wilson-loop functionals, thereby extending the results of $[10,13,12]$.

Following the remarks at the end of Section 2, we know the vacuum expectation value of a multiloop functional when we know the vacuum expectation value of its decomposition into loop networks. So, let us denote the non-overlapping pieces of $\alpha_{1}, \ldots, \alpha_{r}$ by $\gamma_{1}, \ldots, \gamma_{m}, m \geq r$ and the homotopically non-trivial loop by $\gamma$. Then, according to Lemma 4.1, we can write the multiloop functional as a certain linear combination of the states

$$
T_{\Gamma\left(a, N_{x}, N_{y}\right),(\vec{\pi}, \pi), c}=\operatorname{tr}\left[\bigotimes_{k=1}^{m} \pi_{k}\left(h_{\gamma_{k}}(A)\right) \otimes \pi\left(h_{\gamma}(A)\right) \cdot c\right] .
$$

Let $\operatorname{Ar}\left(\gamma_{k}\right)$ denote the area of the surface enclosed by $\gamma_{k}$. According to Lemma 4.2, each $\gamma_{k}$ can be expressed as a certain product of the $n_{k}=\operatorname{Ar}\left(\gamma_{k}\right) / a^{2}$ plaquette loops that are enclosed by $\gamma_{k}$, each of them appearing once and only once, and, due to their nonoverlapping character, these sets of plaquette loops for the different loops $\gamma_{k}$ are mutually 
different, that is, they provide independent integration variables! This is a special feature of two dimensions for the plane and the cylinder.

So, let $\gamma_{k}=\beta_{k, 1} \circ \ldots \circ \beta_{k, n_{k}}$ then due to $\pi_{k}\left(h_{\gamma_{k}}\right)=\pi_{k}\left(h_{\beta_{k, 1}}\right) \ldots \pi_{k}\left(h_{\beta_{k, n_{k}}}\right)$ we end up doing the following basic integral ( $\sigma$ an arbitrary irreducible representation)

$$
I_{\sigma}(\beta):=\int_{G} d \mu(g) \sigma(g), \quad d \mu(g)=\exp \left(-\beta\left[1-\frac{1}{N} \Re \operatorname{tr}(g)\right]\right) d \mu_{H}(g) .
$$

The measure $d \mu$ is conjugation invariant and therefore $I_{\sigma}$ has to be proportional to $\sigma(1)$ according to the lemma of Schur. By taking the trace we see that

$$
I_{\sigma}(\beta)=\sigma(1) J_{\sigma}(\beta), \quad J_{\sigma}(\beta):=\frac{1}{d_{\sigma}} \int_{G} d \mu(g) \chi_{\sigma}(g)
$$

where $\chi_{\sigma}=\operatorname{tr} \sigma$ is the character of the irreducible representation and $d_{\sigma}$ is its dimension.

Since the measure for the loop $\gamma$ is just the Haar measure, the vacuum expectation value of our loop network is non-vanishing only if $\pi$ is the trivial representation 0 . Therefore we get altogether

$$
\chi\left(\Gamma\left(a, N_{x}, N_{y}\right),(\vec{\pi}, \pi), c\right)=\delta_{\pi, 0} \prod_{k=1}^{m}\left[\frac{J_{\pi_{k}}(\beta)}{J_{0}(\beta)}\right]^{n_{k}} d_{c}
$$

where $d_{c}$ is the dimension of the irreducible subspace specified by $c$. Here we have made use of the fact that $c$ is a projector and that in the decomposition into irreducibles of $\bigotimes_{k=1}^{n} \pi_{k}$, the irreducible representation specified by $c$ is contained.

Note that the expression (4.13) is completely insensitive to the size of the lattice due to the fact that the plaquettes are non-interacting. Therefore the thermodynamic limit is already taken. The task of taking the continuum limit now reduces to proving that

$$
\omega(\sigma, \alpha):=\lim _{\beta \rightarrow \infty}\left[\frac{J_{\sigma}(\beta)}{J_{0}(\beta)}\right]^{g_{0}^{2} \operatorname{Ar}(\alpha) \beta}
$$

exists.

The proof goes as follows:

For $\beta \rightarrow \infty$, the integrand of $J_{\sigma}(\beta)$ is concentrated at the identity, so it will be sufficient to calculate the integral for $g$ in a neighbourhood $U$ of the identity. To that effect, write $g=e^{A}$ where $A=t^{I} \tau_{I} \in L(G)$ is in the Lie algebra of $G$ and $t^{I}$ are real parameters in a neighbourhood of zero. We thus have upon inserting $g=1_{N}+A+\frac{1}{2} A^{2}+o\left(A^{3}\right)$

$$
1-\frac{1}{N} \Re \operatorname{tr}(g)=-\frac{1}{2} \operatorname{tr}\left(A^{2}\right)+o\left(A^{3}\right)=\frac{1}{2} \sum_{I=1}^{\operatorname{dim}(G)}\left(t^{I}\right)^{2}+o\left(A^{3}\right)
$$

where the term of first order in $A$ vanishes because it is trace-free $(L(G)$ is semi-simple). We have also used the normalization $\operatorname{tr}\left(\tau_{I} \tau_{J}\right)=-N \delta_{I J}$.

Similarly, we have an expansion for the $\sigma$ th irreducible representation of $G$ given by $\sigma(g)=\sigma(1)+X+\frac{1}{2} X^{2}+o\left(X^{3}\right)$ where $X=t^{I} X_{I}$ is the representation of the Lie algebra element $A$ in the $\sigma$-th irreducible representation. Then we have

$$
\chi_{\sigma}(g)=d_{\sigma}+t^{I} \operatorname{tr}\left(X_{I}\right)+\frac{1}{2} t^{I} t^{J} \operatorname{tr}\left(X_{I} X_{J}\right)+o\left(X^{3}\right) .
$$


According to the Baker-Campbell-Hausdorff formula [7] we have that

$$
\begin{aligned}
& e^{t^{I} \tau_{I}} e^{s^{I} \tau_{I}}=e^{r^{I}(s, t) \tau_{I}} \\
& \quad \text { where } r^{I}(s, t)=s^{I}+t^{I}-\frac{1}{2} f_{J K}^{I} s^{J} t^{K}+o\left(s^{2}, t^{2}, s^{3}, s^{2} t, s t^{2}, t^{3}\right)
\end{aligned}
$$

and where $f_{J K}^{I}$ are the structure constants of the semi-simple Lie algebra of $L(G)$ which therefore are completely skew. Now, the Haar measure can be written [7]

$$
d \mu_{H}\left(e^{t^{I} \tau_{I}}\right)=\frac{d^{\operatorname{dim}(G)} t}{\operatorname{det}\left(\frac{\partial r^{I}(s, t)}{\partial s^{J}}\right)_{s=0}}=\frac{d^{\operatorname{dim}(G)} t}{1+o\left(t^{2}\right)}
$$

since $\operatorname{det}(\partial r / \partial s)_{s=0}=\operatorname{det}\left(1+\frac{1}{2} t^{I} R_{I}+o\left(t^{2}\right)\right)=1+\frac{1}{2} \operatorname{tr}\left(t^{I} R_{I}\right)+o\left(t^{2}\right)=1+o\left(t^{2}\right)$ where $\left(R_{I}\right)_{K}^{J}=f_{I K}^{J}$ is the $I$-th basis vector of $L(G)$ in the adjoint representation which is trace-free.

We now change coordinates $t \rightarrow \sqrt{\beta} t$, insert (4.15), (4.16) and (4.18) into (4.14), write an expansion in $1 / \sqrt{\beta}$ and integrate with the result

$$
J_{\sigma}(\beta)-J_{0}(\beta)=\frac{1}{d_{\sigma} \beta} J_{0}(\beta) \frac{1}{2} \operatorname{tr}\left(\sum_{I=1}^{\operatorname{dim}(G)}\left(X^{I}\right)^{2}\right)+o\left(1 / \beta^{2}\right) .
$$

But $\sum_{I}\left(X_{I}\right)^{2}=-\lambda_{\pi} \pi(1)$ is the Casimir invariant and $\lambda_{\pi}$ its eigenvalue. Therefore we arrive finally at $\omega(\pi, \gamma)=\exp \left(-\frac{1}{2} \lambda_{\pi} g_{0}^{2} \operatorname{Ar}(\gamma)\right)$ and thus

$$
\chi\left(\Gamma\left[\left\{\gamma_{k}\right\}, \gamma\right],(\vec{\pi}, \pi), c\right)=\delta_{\pi, 0} d_{c} \exp \left(-\frac{1}{2} g_{0}^{2} \sum_{k=1}^{m} \lambda_{k} \operatorname{Ar}\left(\gamma_{k}\right)\right) .
$$

\section{Verification of the axioms and comparison with the Hamiltonian formal-}

ism. Let us first verify the axioms.

III) The generating functional (4.20) clearly depends only on the areas of the various loops involved and therefore is not only invariant under the Euclidean group (rather, the symmetry group of the metric on the cylinder) but even under area-preserving diffeomorphisms.

IV) Reflection positivity is also satisfied because after dividing by the space $\mathcal{N}$ of null vectors in $V$ we obtain a scalar product which is positive definite as we will show now by employing the algorithm displayed in [9]:

Consider a multiloop $\left\{\alpha_{1}, \ldots, \alpha_{s}\right\}, s \leq r$, which is composed, among others, of homotopically non-trivial loops. Let $\gamma$ be the horizontal loop at $t=0$ and write every homotopically non-trivial loop $\eta$ occurring in the multiloop $\left\{\alpha_{1}, \ldots, \alpha_{s}\right\}$ as $\eta=[\eta \circ$ $\left.\gamma^{-1}\right] \circ \gamma$ where the loop in brackets is homotopically trivial, thereby obtaining a multiloop $\tilde{\alpha}_{1}, \ldots, \tilde{\alpha}_{s}$ whose homotopically non-trivial contribution comes from $\gamma$ only. It follows that all the vectors in $V$ can be written as linear combinations of loop networks where the homotopically non-trivial contribution comes form $\gamma$. The special feature of the loop $\gamma$ is that it is left invariant under the time reflection operation. We now write

$$
T_{\Gamma,(\vec{\pi}, \pi), c}=T_{\Gamma-\gamma, \vec{\pi}, c_{i}^{j}} \pi_{j}^{i}\left(h_{\gamma}\right)
$$

where $c_{i}^{j}$ is the matrix obtained from $c$ by fixing the last two indices of both its $m+1$ 
fold multi-indices to be $i, j$. Now, observing that for $\Gamma, \Gamma^{\prime}$ supported in the positive time half-space it is true that $\Theta(\Gamma-\gamma), \Gamma^{\prime}-\gamma$ contain only topologically trivial loops enclosing disjoint areas in the two-dimensional spacetime. The non-interactive nature of the measure therefore implies, using the basic integral $\int_{G} d \mu_{H} \bar{\pi}_{j}^{i} \pi_{l}^{\prime k}=1 / d_{\pi} \delta_{\pi, \pi^{\prime}} \delta^{i k} \delta_{j l}$, that

$$
\left\langle\Theta T_{\Gamma,(\vec{\pi}, \pi), c}, T_{\Gamma^{\prime},\left(\vec{\pi}^{\prime}, \pi^{\prime}\right), c^{\prime}}\right\rangle=\overline{\chi\left(\Theta(\Gamma-\gamma), \vec{\pi}, c_{j}^{i}\right)} \chi\left(\Gamma^{\prime}-\gamma, \vec{\pi}^{\prime}, c_{j}^{\prime}{ }^{i}\right) \frac{1}{d_{\pi}} \delta_{\pi, \pi^{\prime}} .
$$

According to (4.20) each of the characteristic functionals on the right-hand side of (5.1) are proportional to $\pi_{j}^{i}(1)$ and the usual trace argument shows, using the fact that $\chi$ is in particular invariant under $\Theta$, that

$$
T_{\Gamma,(\vec{\pi}, \pi), c}-\frac{\chi\left(\Gamma-\gamma, \vec{\pi}, c_{i}^{i}\right)}{\sqrt{d_{\pi}}} \chi_{\pi}\left(h_{\gamma}\right)
$$

is a null vector. Therefore the physical Hilbert space is just given by $\mathcal{H}:=\overline{V / \mathcal{N}}=$ $L_{2}\left(G, d \tilde{\mu}_{H}\right), \tilde{\mu}_{H}$ being the effective measure obtained from $\mu_{H}$ by restricting integration to gauge invariant functions (characters, that is, functions on the Cartan subgroup of $G$ ), which thus leaves us with a positive definite sesquilinear form.

V) We prove ergodicity as follows:

$\gamma(t):=T(t) \gamma$ is the horizontal loop at time $t$. Now let $\alpha(t):=\gamma(t) \circ \gamma^{-1}$, then we have by the representation property

$$
\chi_{\pi}\left(h_{\gamma(t)}\right)=\operatorname{tr}\left(\pi\left(h_{\alpha(t)}\right) \pi\left(h_{\gamma}\right)\right)
$$

so that with respect to $($, ) we have

$$
\chi_{\pi}\left(h_{\gamma(t)}\right)=\frac{1}{d_{\pi}}\left[\int_{\overline{\mathcal{A} / \mathcal{G}}} d \mu \chi_{\pi}(\alpha(t))\right] \chi_{\pi}\left(h_{\gamma}\right)=\exp \left(-\frac{1}{2} g_{0}^{2} \lambda_{\pi} L_{x} t\right) \chi_{\pi}\left(h_{\gamma}\right) .
$$

Therefore $\lim _{t \rightarrow \infty} \frac{1}{t} \int_{0}^{t} d s T(s) \chi_{\pi}=\delta_{0, \pi}=\int d \mu \chi_{\pi}$ and the proof is complete. Meanwhile we see from the definition of the Hamiltonian as the generator of time translations that

$$
\left(\chi_{\pi^{\prime}}, T(t) \chi_{\pi}\right)=\exp \left(-\frac{1}{2} \lambda_{\pi} g_{0}^{2} L_{x} t\right) \delta_{\pi, \pi^{\prime}} \stackrel{!}{=}\left(\chi_{\pi^{\prime}}, \exp (-t H) \chi_{\pi}\right)
$$

and the completeness of the $\chi_{\pi}$ on $L_{2}\left(G, d \tilde{\mu}_{H}\right)$ allows us to conclude that

$$
H=-\frac{g_{0}^{2}}{2} L_{x} \Delta
$$

is the coordinate representation for the Hamiltonian where $\Delta$ is the Casimir operator on $G$. The unique vacuum vector is $\Omega=1$, the only vector annihilated by the Hamiltonian.

We therefore have a simple finite dimensional model in front of us for which the proposed axioms are indeed verified, thus proving non-triviality of the axioms.

Let us now make contact with the Hamiltonian analysis:

After splitting 2-dimensional Minkowski spacetime into space and time, the action for 2-dimensional YM theory becomes [5]

$$
S=\int_{\mathbb{R}} d t \int_{\Sigma} d x\left[\dot{A}_{I} E^{I}-\left[-\Lambda^{I} \mathcal{G}_{I}+\frac{g_{0}^{2}}{2} E^{I} E^{I}\right]\right]
$$

where $\Sigma=\mathbb{R}$ or $S^{1}$. Here $A=A_{x}$ is the (pull-back to $\Sigma$ of the) $G$-connection and $E=$ $\frac{1}{g_{0}^{2}}\left(\partial_{t} A_{x}-\partial_{x} A_{t}+\left[A_{t}, A_{x}\right]\right)$ is its electric field. The Gauss constraint and the Hamiltonian 
are respectively given by (a prime means a derivative with respect to $x$ )

$$
\mathcal{G}_{I}=E_{I}^{\prime}+[A, E]_{I} \text { and } H=\int_{\Sigma} d x \frac{g_{0}^{2}}{2} E^{I} E^{I} .
$$

Now, multiplying the Gauss constraint with $E^{I}$ we infer that the Hamiltonian density is a constant on the constraint surface $\frac{1}{2}\left(E^{I} E^{I}\right)^{\prime}=0$ which immediately implies that the energy is infinite on the plane unless that constant is zero. This in turn implies $E^{I}=0$ and the theory becomes trivial on the plane.

On the cylinder, however, the theory is less trivial, the Hamiltonian is just given by $H=\frac{g_{0}^{2} L_{x}}{2}\left(E^{I} E^{I}\right)$ which remains finite for finite $E$ due to the compactness of the $x$ direction.

We now quantize this theory along the Dirac approach, that is, we solve the constraints by imposing it on the states which we choose in the connection representation.

The canonical commutation relations resulting from the Poisson bracket $\left\{A_{I}(x), E^{J}(y)\right\}=\delta_{I}^{J} \delta(x, y)$ for the canonical pair $A_{I}, E^{I}$ are met if we choose the following operator representation

$$
\left(\hat{A}_{I}(x) \psi\right)(A)=A_{I}(x) \psi(A), \quad\left(\hat{E}_{I}(x) \psi\right)(A)=-i \frac{\delta}{\delta A_{I}(x)} \psi(A) .
$$

Imposing the Gauss constraint on the state space then immediately tells us that they have to be gauge invariant, that is, they have to be built from Wilson loops. But the only loop that we have is $\gamma=\Sigma=S^{1}$ itself, therefore a complete set of physical states is given by the characters $\chi_{\pi}\left(h_{\gamma}(A)\right)$ which form an orthonormal base on $L_{2}\left(G, d \tilde{\mu}_{H}\right)$.

Direct evaluation reveals $\left(g=h_{\gamma}(A)\right)$

$$
\hat{H}(x) \chi_{\pi}(g)=\frac{g_{0}^{2} L_{x}}{2} X_{I}(g) X_{I}(g) \chi_{\pi}(g)=\frac{g_{0}^{2} L_{x}}{2} \Delta(g) \chi_{\pi}(g)=-\frac{g_{0}^{2} L_{x} \lambda_{\pi}}{2} \chi_{\pi}(g)
$$

where $X_{I}(g)=\operatorname{tr}\left(g \tau_{I} \partial / \partial g\right)$ is the left invariant vector field on $G$. This demonstrates exact agreement between both approaches.

Acknowledgements. I am grateful for many important insights obtained in the course of discussions with Abhay Ashtekar, Jurek Lewandowski, Donald Marolf and José Mourão.

The author was supported in part by the NSF Grant PHY93-96246, the Eberly research fund of The Pennsylvania State University and by DOE-Grant DE-FG0294ER25228 to Harvard University.

\section{References}

[1] A. Ashtekar, New Hamiltonian formulation of general relativity, Phys. Rev. D 36 (1987), $1587-1602$.

[2] A. Ashtekar, C. J. Isham, Representation of the holonomy algebras of gravity and nonabelian gauge theories, Classical Quantum Gravity 9 (1992), 1433-1467.

[3] A. Ashtekar, J. Lew andowski, Representation Theory of analytic holonomy $C^{*}$ algebras, in: Knots and quantum gravity, J. Baez (ed.), Oxford University Press, 1994, 21-61. 
[4] A. Ashtekar, J. Lewandowski, D. Marolf, J. Mourão, T. Thiemann, A manifestly gauge invariant approach to quantum theories of gauge fields, in: Geometry of Constrained Dynamical Systems, J. Charap (ed.), Cambridge University Press, Cambridge, 1994, 60-86.

[5] A. Ashtekar, J. Lewandowski, D. Marolf, J. Mourão, T. Thiemann, Euclidean YangMills Theory in two dimensions: A complete solution, Preprint CGPG-95/7-3.

[6] J. Baez, Spin network states in gauge theory, Adv. Math. (in press).

[7] M. Creutz, Quarks, Gluons and Lattices, Cambridge University Press, New York, 1983.

[8] R. Giles, Reconstruction of gauge potentials from Wilson loops, Phys. Rev. D 24 (1981), $2160-2168$.

[9] J. Glimm, A. Jaffe, Quantum Physics, 2nd ed., Springer, New York, 1987.

[10] L. Gross, C. King, A. Sengupta, Two-dimensional Yang-Mills theory via stochastic differential equations, Ann. Physics 194 (1989), 65-112.

[11] S. Helgason, Differential Geometry, Lie Groups and Symmetric Spaces, Academic Press, San Diego, 1978.

[12] V. A. Kazakov, Wilson loop average for an arbitrary contour in two-dimensional $U(N)$ gauge theory, Nuclear Phys. B 179 (1981), 283-292.

[13] S. Klimek, W. Kondracki, A construction of two-dimensional quantum chromodynamics, Comm. Math. Phys. 113 (1987), 389-402.

[14] D. Marolf, J. M. Mourão, On the support of the Ashtekar-Lewandowski measure, Comm. Math. Phys. 170 (1995), 583-606.

[15] V. Rivasseau, From perturbative to constructive renormalization, Princeton University Press, Princeton, 1991.

[16] C. Rovelli, L. Smolin, Spin-networks and quantum gravity, Preprint CGPG-95/4-4.

[17] E. Seiler, Gauge theories as a problem of constructive quantum field theory and statistical mechanics, Lecture Notes in Phys. 159, Springer, Berlin, 1982.

[18] T. Thiemann, A Minlos theorem for gauge theories, in preparation.

[19] T. Thiemann, The inverse loop transform, Preprint CGPG-95/7-1.

[20] T. T. W u, C. N. Yang, Concept of nonintegrable phase factors and global formulation of gauge fields, Phys. Rev. D 12 (1975), 3845-3857.

[21] T. T. Wu, C. N. Yang, Some remarks about unquantized non-abelian gauge fields, Phys. Rev. D 12 (1975), 3843-3844.

[22] Y. Yamasaki, Measures on infinite dimensional spaces, World Scientific, Philadelphia, 1985. 